\title{
ESTUDO REOLÓGICO DE FLUIDOS DE PERFURAÇÃO À BASE DE ÁGUA: INFLUÊNCIA DO TEOR DE SÓLIDOS, VELOCIDADE E TEMPO DE AGITAÇÃo
}

\author{
Luciana Viana Amorim ${ }^{1}$, Cynthia Morais Gomes ${ }^{3}$, Flávio Luiz H. da Silva ${ }^{2}$, \\ Hélio de Lucena Lira ${ }^{1}$ e Heber Carlos Ferreira ${ }^{1}$
}

Recebido em 21/07/2004, aceito em 03/01/2005

\begin{abstract}
RESUMO O objetivo deste trabalho é estudar a influência do teor de sólidos, velocidade e tempo de agitação (fatores de entrada) nos parâmetros reológicos e de filtração de fluidos de perfuração à base de água e argila, utilizando um planejamento fatorial do tipo $2^{3}$ com três experimentos no ponto central + configuração estrela. Foram estudadas duas amostras de bentonitas sódicas industrializadas na Paraíba, caracterizadas por análise química, difração de raio-X e microscopia eletrônica de transmissão. Os fluidos foram preparados com concentrações de 3,0\% a 8,0\% em massa, utilizando as velocidades de $10.000,14.000$ e $17.000 \mathrm{rpm}$ e os tempos de agitação de 7, 10, 15, 20 e 23 min. Através da regressão dos dados experimentais, verificou-se que dentre os fatores avaliados, o teor de sólidos foi a variável que apresentou maior influência estatisticamente significativa sobre as propriedades reológicas dos fluidos.
\end{abstract}

Palavras-Chave: Teor de sólidos, velocidade e tempo de agitação, reologia, fluidos de perfuração.

\begin{abstract}
The aim of this work is to study the influence of the concentration of solids, speed and agitation time on the rheology of water and clay based drilling fluids. Two sodium bentonite clays supplied by industry from Campina Grande, PB were studied. The physical and mineralogical characterization of the samples was done through chemical analysis, X-ray diffraction and transmission electron microscopy. A type $2^{3}$ factorial design with three experiments in the central point + star configuration was developed to evaluate the effect of the some process variables (concentration of solids, speed and agitation time) over the apparent and plastic viscosity and fluid loss. The drilling fluids with concentrations from 3,0\% to $8,0 \% \mathrm{w} / \mathrm{w}$ were prepared and mixed by using the speeds of 10,000,14,000 and 17,000 rpm and agitations time period of 7, 10, 15, 20 and $23 \mathrm{~min}$. The regression analyzis shows that the concentration of solids present correlation with significance with the rheological properties of the fluids.
\end{abstract}

Keywords: Concentration of solids, speed and agitation time, rheology, drilling fluids.

\section{INTRODUÇÃO}

Os fluidos de perfuração, também chamados de lamas, são empregados para auxiliar o processo de perfuração de poços. Atualmente, estão disponíveis diversos tipos de fluidos de perfuração, podendo-se destacar os fluidos à base de água e argila. Esses fluidos vêm sendo utilizados há mais de uma centena de anos (DARLEY E GRAY, 1988) e são empregados na indústria de extração de petróleo, tanto em perfurações terrestres (on-shore) quanto marítimas (off-shore), nas perfurações de poços artesianos, bem como em operações de sondagem.

As argilas bentoníticas têm fundamental importância na composição destes fluidos, pois agem como viscosificante e agente tixotrópico, sendo propriedades necessárias para que estes desempenhem as funções que lhes são requeridas, como limpeza e estabilidade do poço.

Segundo DARLEY E GRAY (1988), as quantidades de argila adicionadas ao fluido variam de acordo com as formações a serem perfuradas; para promover a estabilização de formações instáveis, 70 a $100 \mathrm{~kg} / \mathrm{m}^{3}$ de argila devem ser utilizadas, enquanto que para evitar problemas de perdas de circulação, essa concentração varia de 85 a $110 \quad \mathrm{~kg} / \mathrm{m}^{3}$. Acrescentam ainda, que o estudo do teor de sólidos é de grande importância e de prática comum em campos de perfuração para avaliar o desempenho dos fluidos quando em serviço, servindo como guia para tratamentos de manutenção, indicando a necessidade ou não de diluição do fluido.

$$
\text { ASSUNÇÃO E FERREIRA (1979), }
$$
estudaram o efeito da concentração de argila (5,1\% a 6,9\%), da velocidade (4.000 rpm a $10.000 \mathrm{rpm}$ ) e do tempo de agitação (8 min a 24 min) na reologia de dispersões de argilas esmectíticas industrializadas da Paraíba. VALENZUELA DÍAZ et al. (1982, 1991), apresentaram curvas de viscosidades aparente e plástica em função da concentração de diversos tipos de argila, dentre elas, de argilas bentoníticas, sendo observado que as viscosidades das dispersões variam exponencialmente com a concentração de sólidos. As especificações da PETROBRAS (1998), classificam as argilas bentoníticas para uso como agente viscosificante em fluidos à base de água em: argila do tipo I e argila do tipo II. As argilas do tipo I são aquelas que em dispersão, na

\footnotetext{
${ }^{1}$ Departamento de Eng. de Materiais - Universidade Federal de Campina Grande - UFCG

(luciana@labdes.ufcg.edu.br) (helio@dema.ufcg.edu.br), (heber@dema.ufcg.edu.br)

${ }^{2}$ Departamento de Eng. Química - Universidade Federal de Campina Grande - UFCG (flhs@deq.ufcg.edu.br)

${ }^{3}$ Pós-graduação em Eng. de Materiais, Universidade Federal de Santa Catarina (cynthia@pg.materiais.ufsc.br)
} 
concentração de 4,86\% em massa, apresentam valores mínimos de viscosidades aparente e plástica de 15,0 cP e 4,0 cP, espectivamente, e valor máximo de de volume de filtrado de 18,0 $\mathrm{mL}$. As argilas do tipo II são aquelas que em concentração de $6,40 \%$ em massa apresentam viscosidades mínimas aparente e plástica de 15,0 cP e 6,0 cP, respectivamente, e volume de filtrado máximo de $16,0 \mathrm{~mL}$.

O objetivo deste trabalho é estudar a influência do teor de sólidos, da velocidade e do tempo de agitação na reologia de fluidos de perfuração à base de água e argila, utilizando-se da metodologia do planejamento fatorial.

\section{MATERIAL E MÉTODOS}

Foram estudadas duas amostras de argilas bentoníticas sódicas industrializadas em Campina Grande (PB), proveniente das jazidas localizadas no município de Boa Vista (PB), cedidas pela Empresa Bentonit União Nordeste - BUN e comercialmente conhecidas por Brasgel e Brasgel PA.

\section{Caracterização Química e Mineralógica}

A composição química das argilas foi determinada segundo métodos do Laboratório de Análises Minerais, do Centro de Ciências e Tecnologia da Universidade Federal de Campina Grande, PB (MÉTODOS, 2000).

Os difratogramas das amostras originais e tratadas com etileno glicol foram obtidos com difratômetro Siemens/Brucker, modelo AXS D5005, com radiação $\mathrm{CuK}_{\alpha}(40 \mathrm{kV}, 40 \mathrm{~mA})$.

As micrografias eletrônicas de transmissão foram obtidas com equipamento MET Philips CM 200, operando a 200kV.

\section{Planejamento Fatorial}

Para avaliar a influência das variáveis de entrada (teor de sólidos, velocidade e tempo de agitação) sobre as propriedades reológicas (viscosidades aparente e plástica) e de filtração (volume de filtrado) de fluidos de perfuração à base de água e argila, foi utilizado um planejamento fatorial do tipo $2^{3}$ com três experimentos no ponto central + configuração estrela, totalizando 17 corridas experimentais (BARROS NETO et al., 1996). É importante ressaltar que inicialmente foi utilizado o planejamento fatorial do tipo $2^{3}$ com três experimentos no ponto central, contudo o modelo linear obtido não apresentou resultados satisfatórios. Desta forma, utilizou-se, de acordo com BARROS NETO et al. (1996), um modelo quadrático por meio da ampliação do planejamento, ou seja, fazendo-se uso do planejamento em estrela. Para tanto, foi acrescentado ao planejamento original um outro idêntico, porém girado de $45^{\circ}$ em relação à orientação de partida.

A regressão dos dados experimentais foi realizada utilizando o programa STATISTICA ${ }^{\mathrm{TM}}$ (STATSOFT, ). Os valores codificados e reais das variáveis de entrada empregados no planejamento encontram-se na tabela 1 .

Para o preparo dos fluidos foi utilizado um agitador mecânico da marca Hamilton Beach modelo N5000, que opera com velocidades de 10.000 rpm, 14.000 rpm e 17.000 rpm. Portanto, para as condições de ensaio nas quais as velocidades requeridas, segundo o planejamento fatorial, seriam de $13.500 \mathrm{rpm}$ (ensaios realizados no ponto central) e de $7.600 \mathrm{rpm}$ e $20.000 \mathrm{rpm}$ (ensaios realizados para os níveis -1,682 e $+1,682$ ) foram utilizadas as velocidades de $14.000 \mathrm{rpm}$ e de $17.000 \mathrm{rpm}$, respectivamente.

\section{Preparação dos Fluidos de Perfuração}

Os fluidos de perfuração à base de água deionizada e argila bentonítica nas concentrações de $3,0 \%, 4,0 \%, 5,5 \%, 7,0 \%$ e $8,0 \%$ em massa foram preparados utilizando o planejamento fatorial apresentado na Tabela 1. As velocidades e os tempos de agitação estudados foram de $10.000 \mathrm{rpm}, 14.000 \mathrm{rpm}$ e $17.000 \mathrm{rpm}$ e de $7 \mathrm{~min}$, $10 \mathrm{~min}, 15 \mathrm{~min}, 20 \mathrm{~min}$ e $23 \mathrm{~min}$, respectivamente. As dispersões, após preparação, permaneceram em repouso por um período de 24 h em câmara úmida com 100\% de umidade relativa.

Tabela 1 - Níveis codificados e valores reais das variáveis de entrada do planejamento fatorial do tipo $2^{3}$ com três experimentos no ponto central + configuração estrela.

\section{Estudo Reológico}

Foram determinadas as viscosidades aparente (VA) e plástica (VP) em viscosímetro Fann modelo 35A e o volume do filtrado (VF), em filtro prensa Fann, segundo norma da PETROBRAS (1998a).

\section{RESULTADOS E DISCUSSÃO \\ Caracterização Física e Mineralógica}

$\mathrm{Na}$ tabela 2, estão apresentadas as composições químicas das argilas Brasgel e Brasgel PA.

As amostras apresentaram valores de perda ao rubro (PR) bastante diferenciados, sendo o 
apresentado pela amostra Brasgel duas vezes superior ao da amostra Brasgel PA. A PR representa a perda de água intercalada, a perda de água de hidroxilas dos argilominerais, matéria orgânica e carbonatos (SOUZA SANTOS, 1992). Os teores de $\mathrm{Fe}_{2} \mathrm{O}_{3}$, da ordem de $7,0 \%$, são típicos das argilas do Município de Boa Vista e, provavelmente, provenientes do reticulado cristalino da ilita, que contém cerca de $4 \%$ a $6 \%$ de $\mathrm{Fe}_{2} \mathrm{O}_{3}$, e dos argilominerais do grupo da esmectita, ou seja, montmorilonita ou membro da série isomórfica nontronita-beidelita (SOUZA SANTOS, 1992).

\begin{tabular}{cccccc}
\hline Variáveis & \multicolumn{5}{c}{ Níveis codificados } \\
\cline { 2 - 6 } de & Nível & Nível & Ponto central & Nível & Nível \\
entrada & $-1,682$ & -1 & 0 & +1 & $+1,682$ \\
\hline Teor de sólidos (g) & 3,0 & 4,0 & 5,5 & 7,0 & 8,0 \\
Velocidade de agitação (rpm) & 10.000 & 10.000 & 14.000 & 17.000 & 17.000 \\
Tempo de agitação (min) & 7 & 10 & 15 & 20 & 23 \\
\hline
\end{tabular}

Tabela 2 - Composição química das amostras Brasgel e Brasgel PA.

\begin{tabular}{ccc}
\hline Componentes (\%) & Brasgel & Brasgel PA \\
\hline Perda ao Rubro - PR & 18,37 & 8,59 \\
Resíduos Insolúveis - RI & 2,14 & 1,49 \\
$\mathrm{SiO}_{2}$ & 51,69 & 55,46 \\
$\mathrm{Fe}_{2} \mathrm{O}_{3}$ & 7,19 & 7,88 \\
$\mathrm{Al}_{2} \mathrm{O}_{3}$ & 18,76 & 22,00 \\
$\mathrm{CaO}$ & Traços & Traços \\
$\mathrm{MgO}$ & Traços & 3,00 \\
$\mathrm{Na} O$ & 1,50 & 1,00 \\
$\mathrm{~K}_{2} \mathrm{O}$ & 0,23 & 0,50 \\
Total & 99,88 & 99,92 \\
\hline
\end{tabular}

Comparando os teores apresentados para os óxidos de cálcio $(\mathrm{CaO})$ e magnésio $(\mathrm{MgO})$, observou-se que a argila Brasgel PA apresentou teor de $\mathrm{MgO}$ de 3,00\%, diferenciando-a da argila Brasgel. A presença deste óxido indica que as argilas Brasgel e Brasgel PA foram produzidas a partir de diferentes variedades mineralógicas. Provavelmente, foi utilizada para a produção da argila Brasgel PA uma variedade mineralógica mais nobre, que apresenta teores mais elevados de $\mathrm{MgO}$, conferindo ao fluido maior viscosidade aparente e menor volume de filtrado. Segundo AMORIM E PEREIRA (2003), as variedades mineralógicas mais nobres de Boa Vista, PB encontram-se praticamente esgotadas. Estas são, provavelmente, utilizadas apenas na produção de bentonitas sódicas para finalidades industriais que requerem melhor comportamento reológico, como para uso nos fluidos de perfuração.

Em recentes estudos realizados por AMORIM (2003) foi observado que a argila Brasgel PA apresenta maior valor de viscosidade aparente e menores valores de viscosidade plástica e volume de filtrado quando comparada a Brasgel. Os dados foram obtidos com a argila em dispersão na concentração de 4,86 \%, seguindo as normas da PETROBRAS (1998a).

$\mathrm{Na}$ Figura 1, estão apresentados os difratogramas das amostras Brasgel e Brasgel PA com e sem o tratamento com etileno glicol. As amostras Brasgel e Brasgel PA apresentaram difratogramas típicos de argilas bentoníticas, com presença de argilominerais do grupo da esmectita, confirmada pela mudança do seu pico característico após tratamento com etileno glicol, de $14-15 \AA$ para $17 \AA$. As amostras apresentaram ainda pico a $3,35 \AA$, característico da presença de quartzo.

Na Figura 2, estão apresentadas as micrografias das amostras Brasgel e Brasgel PA, que apresentaram aspecto típico das argilas do grupo da esmectita, com partículas de perfil irregular, algumas com contorno mal definido e grande tendência ao enrolamento devido à pequena espessura das placas examinadas, além de elevada tendência para agregação em virtude do desbalanceamento de cargas. As micrografias apresentaram fundo difuso causado pela dispersão de agregados de pequena espessura. 


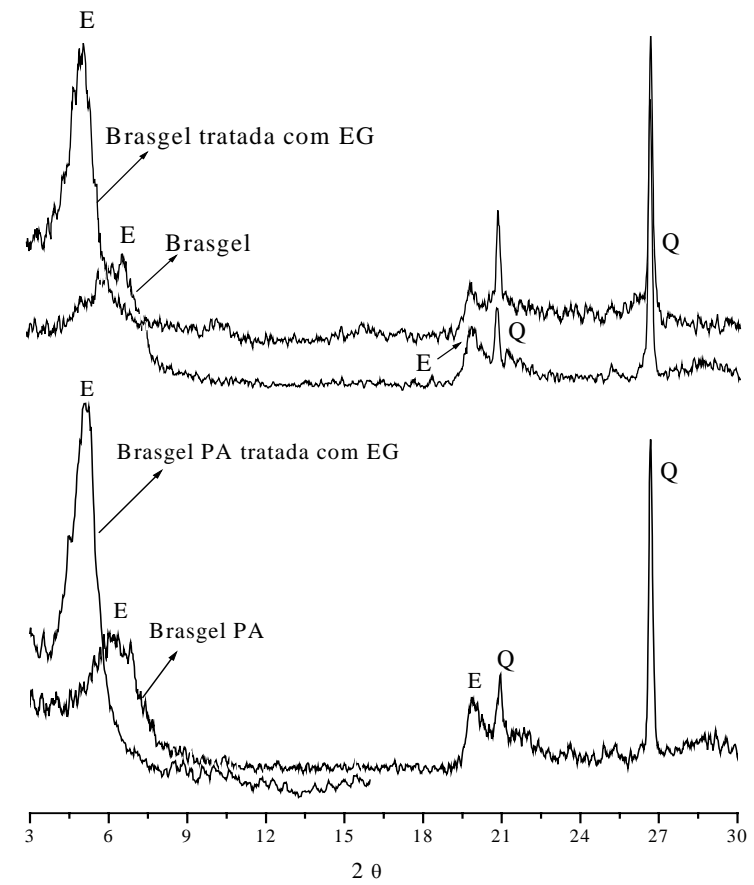

Legenda:

E - Esmectita

Q - Quartzo

Fr. Ftilenn olirnl

Figura 1 - Difratogramas das amostras de argilas bentoníticas Brasgel e Brasgel PA.

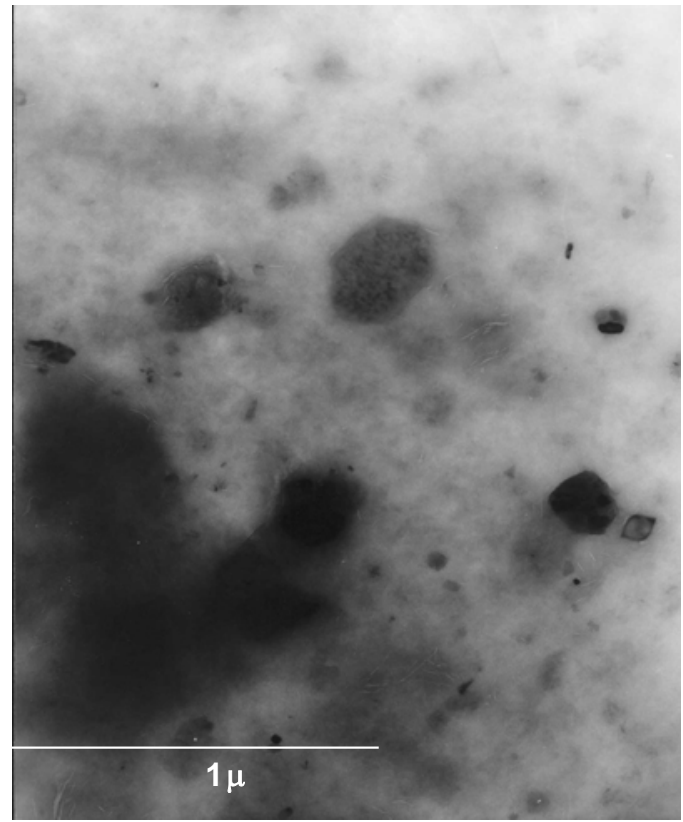

(a)

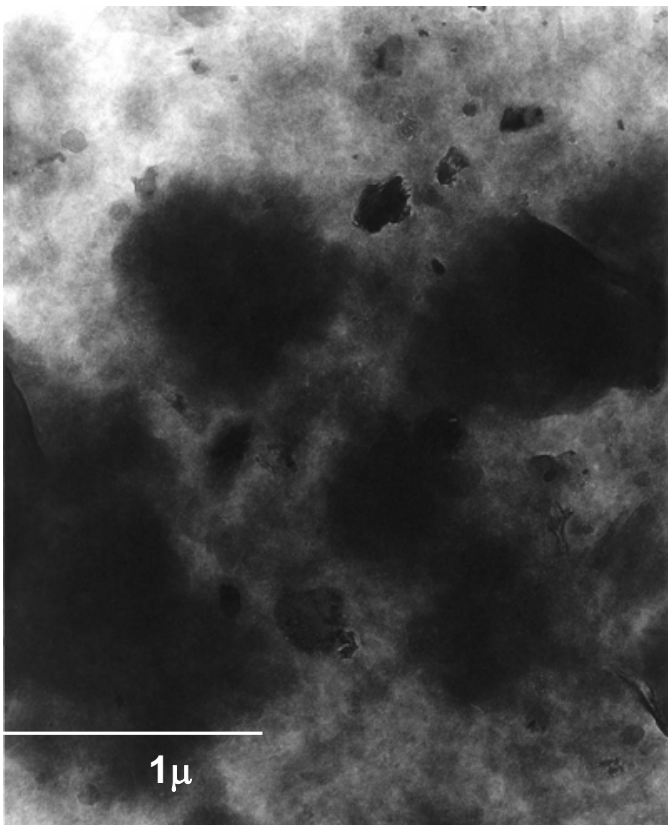

(b)

Figura 2 - Micrografias das amostras (a) Brasgel e (b) Brasgel PA

\section{Estudo Reológico}

Nas tabelas 3 e 4, encontram-se a matriz de planejamento fatorial e os resultados de viscosidade aparente (VA), viscosidade plástica (VP) e volume de filtrado (VF) obtidos com os fluidos preparados com as amostras Brasgel e Brasgel PA, respectivamente.
Na Tabela 5, estão apresentadas as análises de variância e os modelos matemáticos codificados para as propriedades reológicas (VA, $\mathrm{VP}$ ) e para o volume de filtrado (VF) dos fluidos de perfuração estudados.

A análise de significância estatística mostrou que os coeficientes de correlação (R) e 
as porcentagens de variação explicada dos resultados experimentais para VA, VP e VF dos fluidos preparadas com as amostras Brasgel e Brasgel PA foram satisfatórios, superiores a 0,98 e 96,0\%, respectivamente, com exceção dos apresentados para VP dos fluidos preparados com a argila Brasgel PA. É importante ressaltar que estes resultados são significativos para o nível de $95,0 \%$ de confiança.

Tabela 3 - Matriz de planejamento fatorial e propriedades reológicas (viscosidades aparente (VA) e plástica (VP)) e de filtração (volume de filtrado (VF)) dos fluidos preparados com a amostra Brasgel.

\begin{tabular}{|c|c|c|c|c|c|c|}
\hline Experimentos & $\begin{array}{c}\text { Teor } \\
\text { de sólidos } \\
(\mathrm{T})\end{array}$ & $\begin{array}{l}\text { Velocidade } \\
\text { de agitação } \\
\text { (v) }\end{array}$ & $\begin{array}{l}\text { Tempo } \\
\text { de agitação } \\
\text { (t) }\end{array}$ & $\begin{array}{l}\text { VA } \\
\text { (cP) }\end{array}$ & $\begin{array}{l}\mathrm{VP} \\
(\mathrm{cP})\end{array}$ & $\begin{array}{l}\mathrm{VF} \\
(\mathrm{mL})\end{array}$ \\
\hline 1 & -1 & -1 & -1 & 3,0 & 3,5 & 24,5 \\
\hline 2 & +1 & -1 & -1 & 12,3 & 5,0 & 17,5 \\
\hline 3 & -1 & +1 & -1 & 3,5 & 3,5 & 24,5 \\
\hline 4 & +1 & +1 & -1 & 13,3 & 6,5 & 17,5 \\
\hline 5 & -1 & -1 & +1 & 3,8 & 3,0 & 25,0 \\
\hline 6 & +1 & -1 & +1 & 12,8 & 6,5 & 18,0 \\
\hline 7 & -1 & +1 & +1 & 4,8 & 3,5 & 25,0 \\
\hline 8 & +1 & +1 & +1 & 14,8 & 5,5 & 16,0 \\
\hline 9 & $-1,682$ & 0 & 0 & 1,8 & 1,5 & 29,5 \\
\hline 10 & $+1,682$ & 0 & 0 & 21,5 & 6,5 & 15,5 \\
\hline 11 & 0 & -1 & 0 & 6,3 & 4,5 & 20,0 \\
\hline 12 & 0 & +1 & 0 & 8,3 & 4,0 & 20,5 \\
\hline 13 & 0 & 0 & $-1,682$ & 6,8 & 5,0 & 20,0 \\
\hline 14 & 0 & 0 & $+1,682$ & 8,3 & 5,0 & 20,5 \\
\hline 15 & 0 & 0 & 0 & 7,8 & 5,0 & 19,5 \\
\hline 16 & 0 & 0 & 0 & 7,3 & 4,5 & 19,0 \\
\hline 17 & 0 & 0 & 0 & 7,5 & 5,0 & 20,0 \\
\hline \multirow{2}{*}{\multicolumn{2}{|c|}{$\begin{array}{l}\text { Valores especificados } \\
\text { (PETROBRAS, 1998) }\end{array}$}} & \multicolumn{2}{|c|}{ Argila tipo I } & $\geq 15,0$ & $\geq 4,0$ & $\leq 18,0$ \\
\hline & & \multicolumn{2}{|c|}{ Argila tipo II } & $\geq 15,0$ & $\geq 6,0$ & $\leq 16,0$ \\
\hline
\end{tabular}

Tabela 4 - Matriz de planejamento fatorial e propriedades reológicas (viscosidades aparente (VA) e plástica (VP)) e de filtração (volume de filtrado (VF)) dos fluidos preparados com a amostra Brasgel PA.

\begin{tabular}{|c|c|c|c|c|c|c|}
\hline Experimentos & $\begin{array}{c}\text { Teor } \\
\text { de sólidos } \\
(\mathrm{T})\end{array}$ & $\begin{array}{l}\text { Velocidade } \\
\text { de agitação } \\
\text { (v) }\end{array}$ & $\begin{array}{c}\text { Tempo } \\
\text { de agitação } \\
\text { (t) }\end{array}$ & $\begin{array}{l}\mathrm{VA} \\
\text { (cP) }\end{array}$ & $\begin{array}{l}\mathrm{VP} \\
(\mathrm{cP})\end{array}$ & $\begin{array}{l}\mathrm{VF} \\
(\mathrm{mL})\end{array}$ \\
\hline 1 & -1 & -1 & -1 & 7,5 & 4,5 & 20,0 \\
\hline 2 & +1 & -1 & -1 & 28,5 & 4,0 & 15,0 \\
\hline 3 & -1 & +1 & -1 & 7,8 & 4,0 & 18,5 \\
\hline 4 & +1 & +1 & -1 & 33,5 & 3,5 & 13,5 \\
\hline 5 & -1 & -1 & +1 & 7,5 & 4,0 & 21,0 \\
\hline 6 & +1 & -1 & +1 & 30,5 & 5,0 & 15,0 \\
\hline 7 & -1 & +1 & +1 & 8,0 & 4,0 & 19,5 \\
\hline 8 & +1 & +1 & +1 & 38,3 & 3,5 & 14,0 \\
\hline 9 & $-1,682$ & 0 & 0 & 4,3 & 3,0 & 23,5 \\
\hline 10 & $+1,682$ & 0 & 0 & 53,3 & 4,0 & 13,0 \\
\hline 11 & 0 & -1 & 0 & 16,5 & 4,5 & 16,5 \\
\hline 12 & 0 & +1 & 0 & 18,3 & 5,0 & 16,5 \\
\hline 13 & 0 & 0 & $-1,682$ & 15,8 & 5,0 & 17,0 \\
\hline 14 & 0 & 0 & $+1,682$ & 18,8 & 5,0 & 15,5 \\
\hline 15 & 0 & 0 & 0 & 16,8 & 4,0 & 15,5 \\
\hline 16 & 0 & 0 & 0 & 17,3 & 4,5 & 15,5 \\
\hline 17 & 0 & 0 & 0 & 17,0 & 4,5 & 15,5 \\
\hline \multirow{2}{*}{\multicolumn{2}{|c|}{$\begin{array}{l}\text { Valores especificados } \\
\text { (PETROBRAS, 1998) }\end{array}$}} & \multicolumn{2}{|c|}{ Argila tipo I } & $\geq 15,0$ & $\geq 4,0$ & $\leq 18,0$ \\
\hline & & \multicolumn{2}{|c|}{ Argila tipo II } & $\geq 15,0$ & $\geq 6,0$ & $\leq 16,0$ \\
\hline
\end{tabular}


Tabela 5 - Análises de variância (ANOVA) e modelos matemáticos codificados das viscosidades aparente (VA) e plástica (VP) e do volume de filtrado (VF) dos fluidos preparados com as amostras Brasgel e Brasgel PA para o planejamento fatorial empregado.

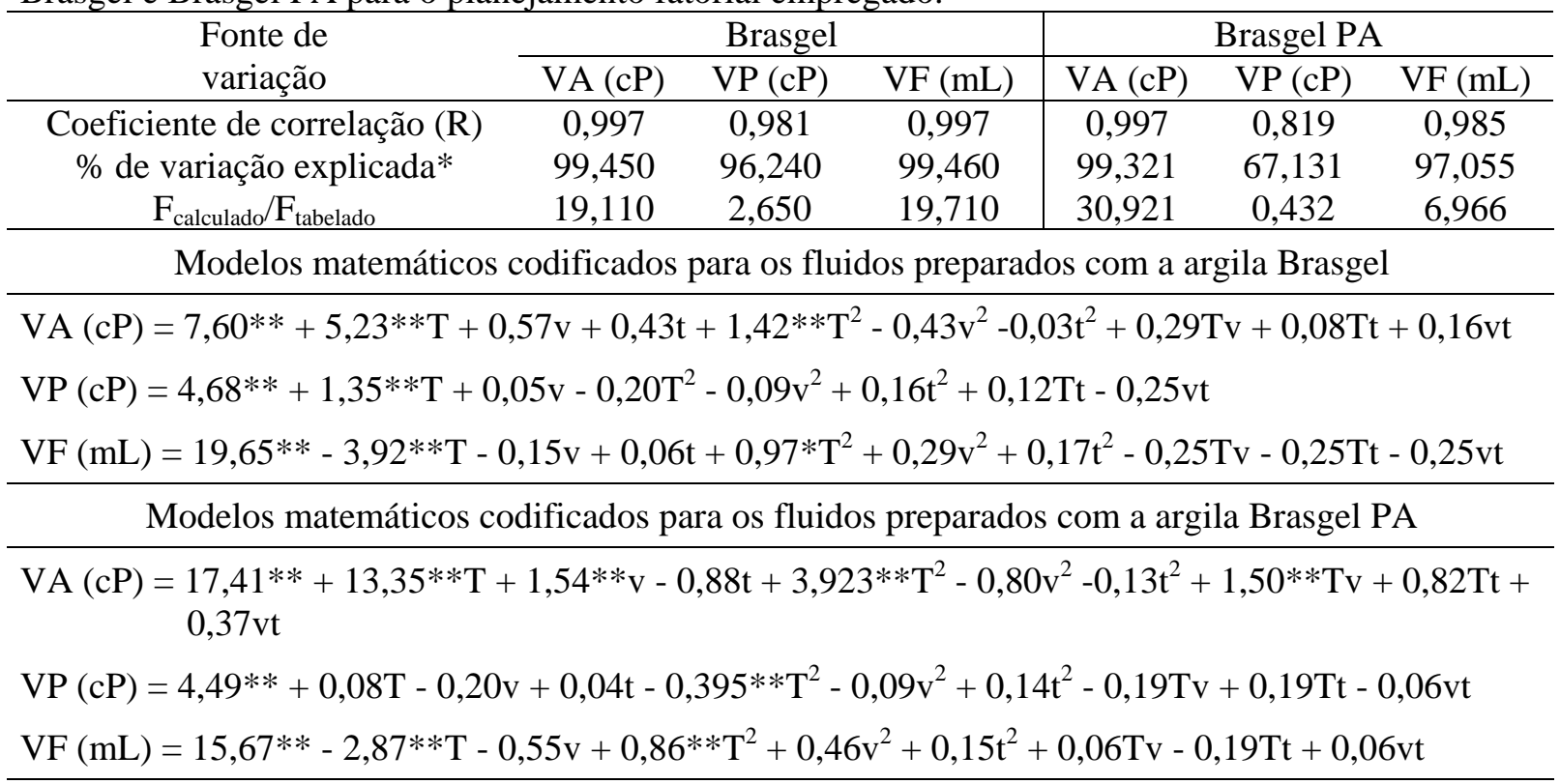

Sendo: $S Q_{R}$ a soma quadrática da regressão, $S Q_{T}$ a soma quadrática total, $\mathrm{T}$ o teor de sólidos, $\mathrm{v}$ a velocidade de agitação e t o tempo de agitação.

$* R^{2}=\left(\frac{S Q_{R}}{S Q_{T}}\right) x 100$

** Estatisticamente significativos ao nível de 95,0 \% de confiança.

As Figuras 3 e 4 apresentam as superfícies de resposta, utilizando-se dos modelos matemáticos codificados apresentados na Tabela 5, para os fluidos preparados com as argilas Brasgel e Brasgel PA, respectivamente. Observou-se que as propriedades reológicas e de filtração dos fluidos estudados são influenciadas pelo teor de sólidos (com exceção da VP dos fluidos preparados com a argila Brasgel PA), ou seja, o aumento na concentração de argila de 3,0\% a 8,0\% interfere de forma estatisticamente significativa nas viscosidades aparente e plástica e no volume de filtrado, ao nível de 95,0\% de confiança, sendo obtidos maiores valores de VA e VP e menores valores de VF. O aumento nos valores de VA e VP decorre da maior intensidade das interações elétricas e de massa entre partículas, que se tornam predominantes com o aumento da concentração de argila. Essas interações promovem a formação de reticulados mais ou menos rígidos, que retêm as moléculas de água diminuindo assim a quantidade de água livre no sistema, e, por conseguinte, o volume de filtrado.

De acordo com STEFAN (1966), a viscosidade plástica é uma variável dependente da interação dos sólidos presentes e/ou uma medida da fricção resultante do choque de uma partícula com outra, sendo influenciada pelo grau de hidratação e pelo campo elétrico resultante das forças repulsivas nas partículas de argila carregadas negativamente. Desta forma, o aumento na concentração de sólidos conduz a maiores valores de VP, como observado para os fluidos preparados com a argila Brasgel. 


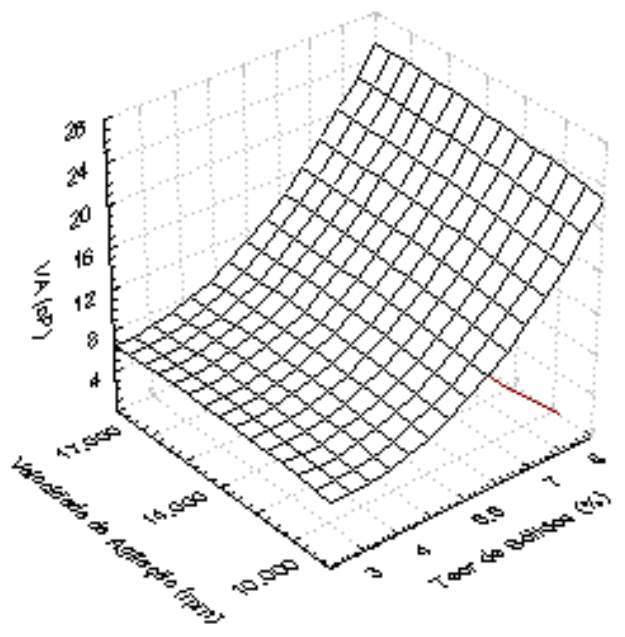

(a)

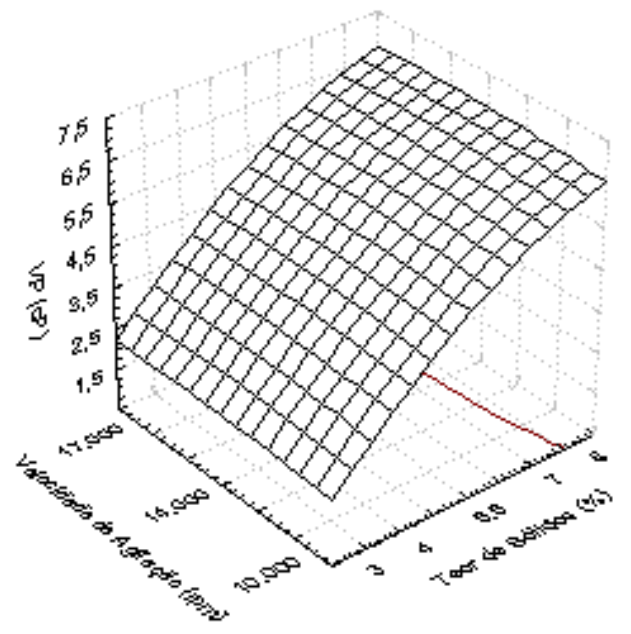

(c)

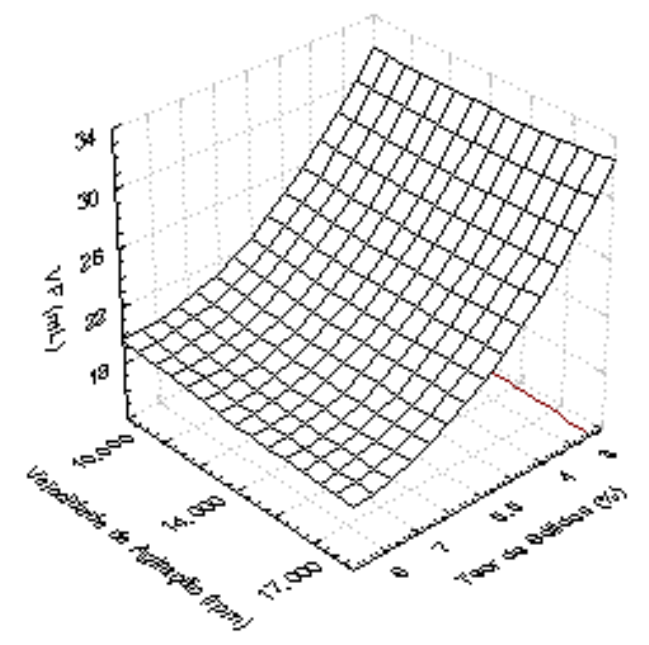

(e)

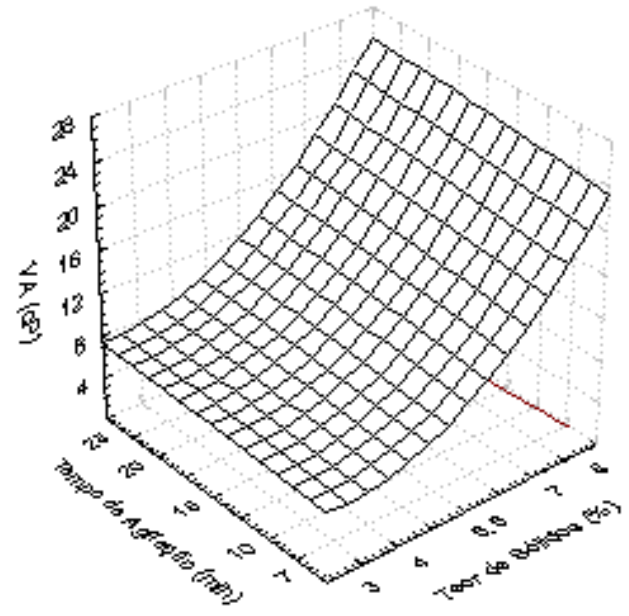

(b)

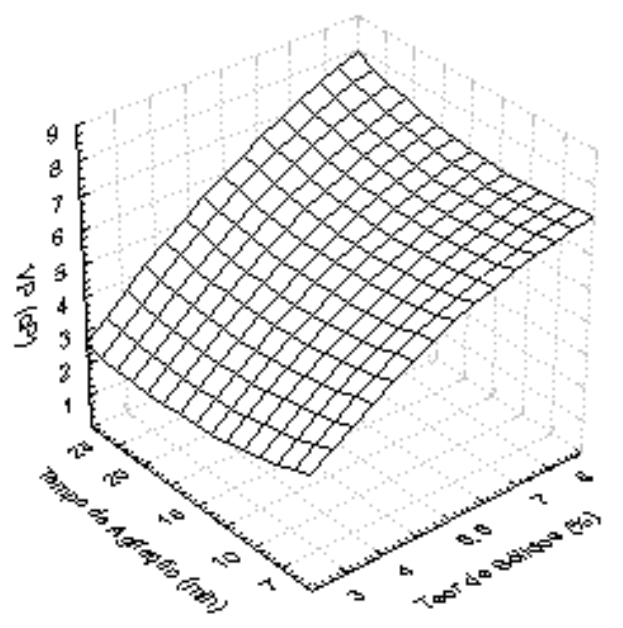

(d)

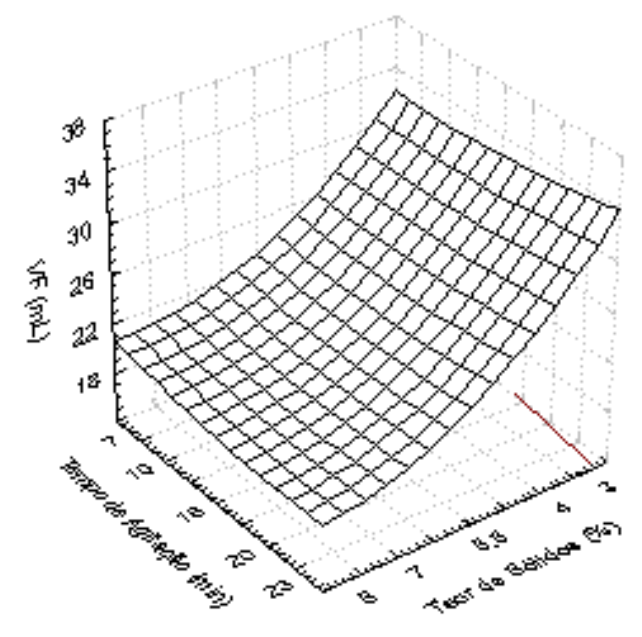

(f)

Figura 3 - Superfícies de resposta para a viscosidade aparente (VA) - (a) e (b), viscosidade plástica (VP) - (c) e (d) e volume de filtrado (VF) - (e) e (f) dos fluidos de perfuração preparados com a argila Brasgel. 


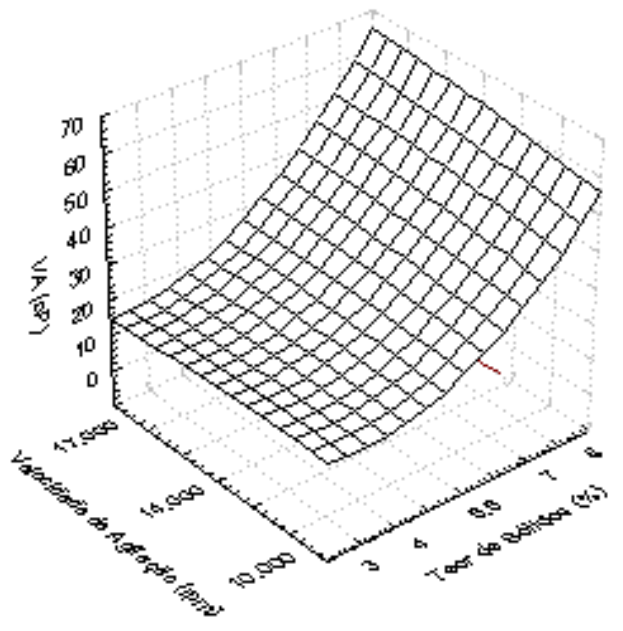

(a)

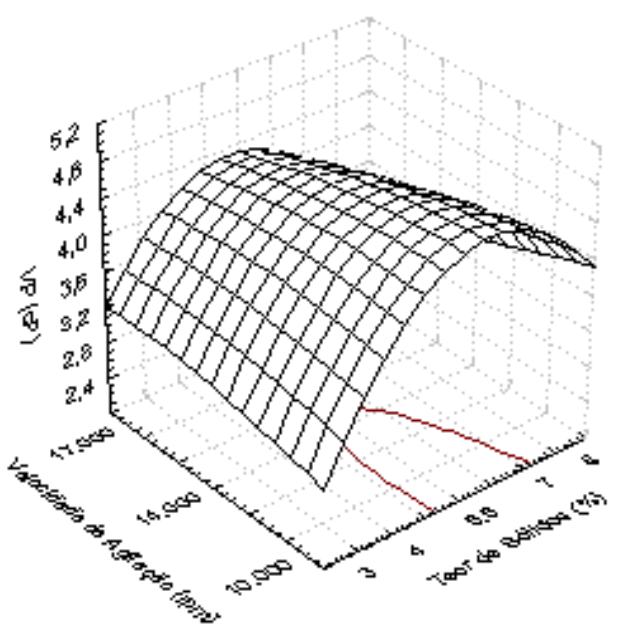

(c)

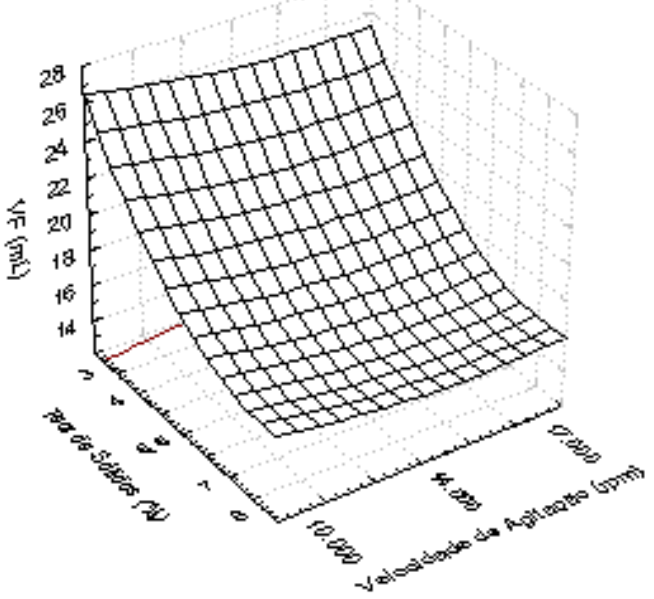

(e)

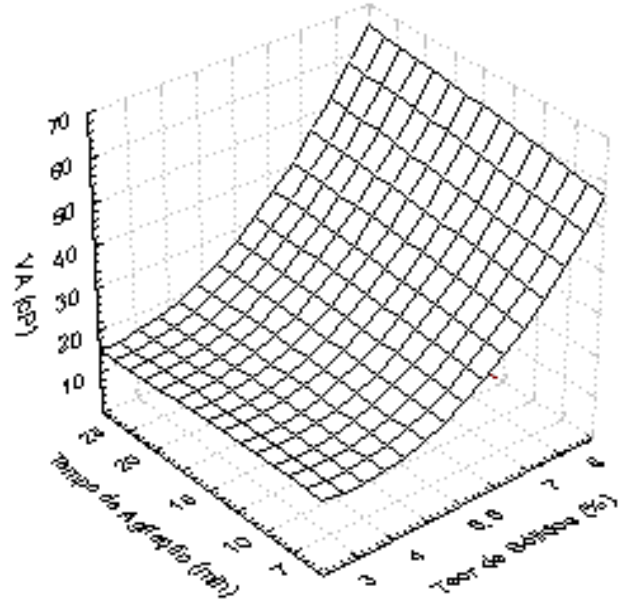

(b)

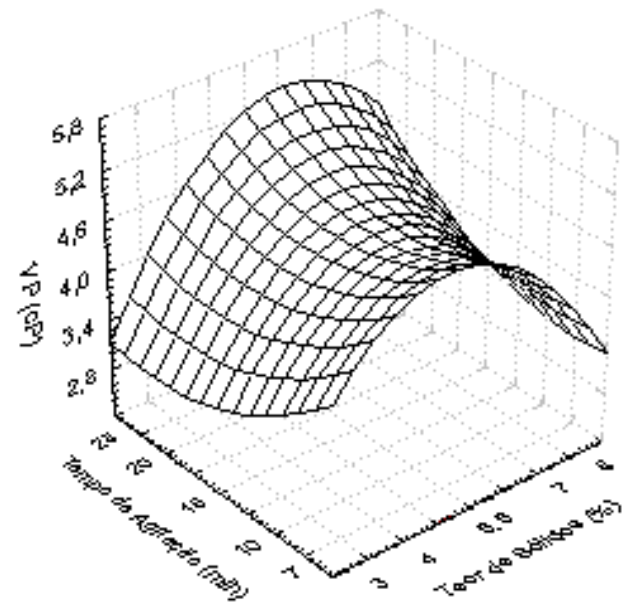

(d)

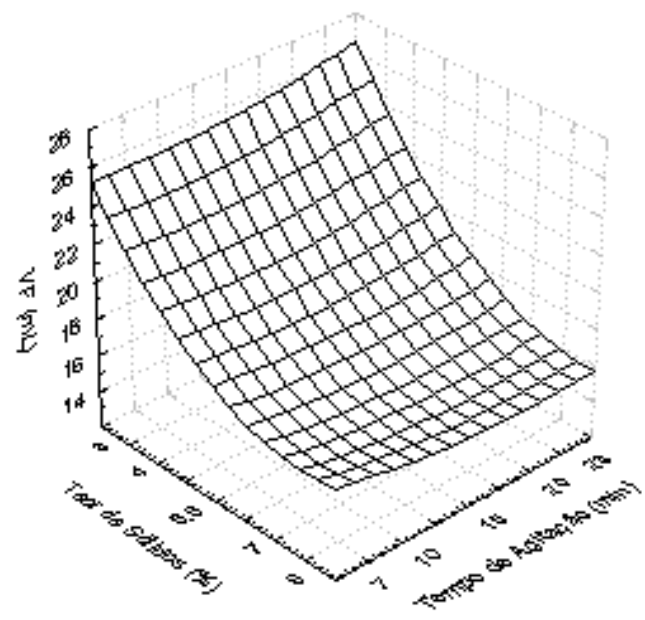

(f)

Figura 4 - Superfícies de resposta para a viscosidade aparente (VA) - (a) e (b), viscosidade plástica (VP) - (c) e (d) e volume de filtrado (VF) - (e) e (f) dos fluidos de perfuração preparados com a argila Brasgel PA 
Segundo DARLEY E GRAY (1988), como as partículas de argila presentes nos fluidos de perfuração são anisométricas e podem formar uma estrutura de gel mesmo a baixas concentrações de sólidos em virtude das interações entre as forças de repulsão e de atração, seu comportamento reológico é altamente dependente da taxa de cisalhamento; a baixas taxas de cisalhamento as partículas de argilas permanecem influenciadas por essas forças e, como conseqüência, sua viscosidade é relativamente alta, contudo, o aumento na taxa de cisalhamento, conduz ao alinhamento das partículas de argila na direção do fluxo e sua viscosidade se torna largamente dependente da concentração de sólidos. A partir dessa teoria, era esperado que os parâmetros reológicos, principalmente a VP, dos fluidos estudados fossem influenciados pelo aumento da velocidade de agitação, contudo, apenas a VA dos fluidos preparados com a argila Brasgel PA foi influenciada pela velocidade de agitação, como mostram os modelos matemáticos codificados, contidos na Tabela 5. Para os demais fluidos não foram observados efeitos significativos sobre a VA, VP e VF quando do aumento da velocidade de agitação de 10.000 rpm a 17.000 rpm.

As superfícies de resposta (Figuras 3 e 4) e a análise de variância (Tabela 5) mostraram ainda que nenhum dos termos envolvendo o tempo de agitação é estatisticamente significativo. Em outras palavras, os parâmetros reológicos e de filtração avaliados não são influenciados pelo aumento do tempo de agitação de $7 \mathrm{~min}$ a $23 \mathrm{~min}$, quando do preparo dos fluidos de perfuração. Comportamento semelhante a este foi observado por AMORIM et al. (2002), que estudaram a influência do tipo de ferramenta, velocidade e tempo de agitação sobre a reologia de dispersões de quatro amostras de argilas bentoníticas industrializadas da Paraíba.

Através da razão entre o teste $\mathrm{F}_{\text {calculado }} \mathrm{e} o$ teste $\mathrm{F}_{\text {tabelado, }}$ apresentada na tabela 5 , observou-se que os modelos matemáticos obtidos para a VA e VF dos fluidos preparados com a argila Brasgel, além de estatisticamente significativos, são altamente preditivos por apresentarem valores superiores a 10,0. Para VP, essa razão foi de 2,65 (superior a 1,0), sendo considerado estatisticamente significativo (BARROS NETO et al., 1996). Para os fluidos preparados com a argila Brasgel PA, o modelo matemático obtido para VA, além de estatisticamente significativo, é altamente preditivo, enquanto que o obtido para VF é estatisticamente significativo.
Para os fluidos preparados com a argila Brasgel, observou-se que o aumento no teor de sólidos de 3,0\% a 8,0\% promoveu acréscimo em VA para qualquer velocidade e tempo de agitação, sendo obtidos valores superiores a 21,0 cP (Figuras 3(a) e 3(b)). Nas Figuras 3(c) e 3(d), observou-se comportamento semelhante ao anterior, sendo obtidos valores de VP de 5,0 cP e através das Figuras 3(e) e 3(f), verificou-se que menores valores de $\mathrm{VF}$, da ordem de 15,0 mL, são obtidos com o aumento no teor de sólidos, também para qualquer velocidade e tempo de agitação. Portanto, esse comportamento evidenciou que o teor de sólidos apresentou influência estatisticamente significativa sobre VA, VP e VF, bem como que os fluidos, quando preparados com concentração de sólidos igual ou superior a 7,0 \%, para quaisquer velocidade e tempo de agitação, apresentaram valores de VP e VF de acordo com as especificações da PETROBRAS (1998), para uso como agente viscosificante em fluidos de perfuração de poços de petróleo à base de água. Contudo, é importante ressaltar que os valores normatizados pela Petrobras devem ser obtidos para fluidos com concentração de argila ativada de 4,86\%, para o tipo I, e de 6,40\%, para o tipo II.

Para os fluidos preparados com a argila Brasgel PA, observou-se que o aumento no teor de sólidos de 3,0\% a 8,0\% promoveu acréscimo em VA para qualquer tempo de agitação, sendo obtidos valores superiores a 50,0 cP (Figuras 4(a) e 4(b)). Nas Figuras 4(c) e 4(d), observou-se que, embora VP não seja estatisticamente influenciada pela concentração de sólidos, maiores valores são obtidos com teores próximos do ponto central (5,5\%), entre 4,0 e 5,0 cP, para qualquer velocidade e tempo de agitação. Por fim, através das Figuras 4(e) e 4(f), verificou-se que menores valores de VF, da ordem de 13,0 mL, são obtidos com o aumento no teor de sólidos, também para quaisquer velocidade e tempo de agitação. Esse comportamento mostra que concentrações de argila próximas de 8,0\% conduzem a fluidos com característica de um sistema floculado-gel, ou seja, com valores de VA muito altos e valores de VP baixos. Como conseqüência desse tipo de sistema, têm-se fluidos com elevados limites de escoamento, o que não é desejável, pois provocam problemas nas etapas iniciais do bombeamento durante as operações de perfuração de poços. Outra importante constatação, é que reologias adequadas, com valores de VA, VP e VF de acordo com as especificações da PETROBRAS (1998) para argila do tipo I, foram obtidas com teores próximos de 5,0\%, podendo- 
se então afirmar que o uso excessivo na concentração de argila, além de implicar em um aumento nos custos da operação de perfuração conduz a fluidos com reologias inadequadas.

Comparando os resultados apresentados pelos fluidos preparados com as argilas Brasgel e Brasgel PA, observou-se que essa última apresenta melhor desempenho, atendendo as especificações da Petrobras (1998) para argilas do tipo I para uso como agente viscosificante e tixotrópico em fluidos de perfuração à base de água. Este comportamento deve-se provavelmente à composição da mistura utilizada para a produção da argila Brasgel PA, como já mencionado. É importante ressaltar, que esta análise foi fundamentada no fato de que as amostras estudadas são provenientes do mesmo fabricante e sob as mesmas condições de processamento.

\section{CONCLUSÕES}

Com o objetivo de estudar a influência do teor de sólidos, da velocidade e do tempo de agitação sobre os parâmetros reológicos e de filtração de fluidos de perfuração à base de água e argila, utilizando-se da metodologia do planejamento fatorial, concluiu-se que:

- as amostras Brasgel e Brasgel PA são compostas por argilominerais do grupo da esmectita e minerais de quartzo;

- o teor de sólidos influencia estatisticamente as propriedades reológicas e de filtração dos fluidos de perfuração, ao nível de 95,0 \% de confiança, sendo obtidos maiores valores de VA e VP e menores valores de VF com o aumento da concentração de argila de 3,0\% a $8,0 \%$;

- $\quad$ as variáveis velocidade e tempo de agitação não apresentaram influência estatisticamente significativa sobre as propriedades reológicas dos fluidos;

- para os fluidos preparados com a argila Brasgel, os modelos matemáticos obtidos para VA e VF, além de estatisticamente significativos, são altamente preditivos, enquanto que o obtido para VP é estatisticamente significativo;

- para os fluidos preparados com a argila Brasgel PA, o modelo matemático obtido para VA é, além de estatisticamente significativo, altamente preditivo, enquanto que o obtido para VF é estatisticamente significativo;

- os fluidos preparados com a argila Brasgel com concentração de sólidos igual ou superior a 7,0 \%, para quaisquer velocidade e tempo de agitação, apresentam valores de VP e VF de acordo com as especificações da PETROBRAS (1998) para uso em perfuração de poços de petróleo;

- os fluidos preparados com a argila Brasgel PA, com teores próximos de 5,0\%, apresentaram reologias adequadas, com valores de VA, VP e VF de acordo com as especificações da PETROBRAS (1998) para argila do tipo I e

- a argila Brasgel PA apresentou melhor desempenho, muito provavelmente, em virtude do uso de variedades mineralógicas mais nobre na composição da mistura utilizada para a sua produção.

\section{AGRADECIMENTOS}

Os autores agradecem à Agência Nacional do Petróleo - ANP, à FINEP, ao MCT, ao $\mathrm{CNPq}$ e ao $\mathrm{CNPq} / \mathrm{CTPETRO}$ pelo suporte financeiro para o desenvolvimento deste trabalho.

\section{REFERÊNCIAS}

AMORIM, L.V. Melhoria, proteção e recuperação da reologia de fluidos hidroargilosos para uso na perfuração de poços de petróleo, 2003. Tese (Doutorado em Engenharia de Processos). Universidade Federal de Campina Grande - UFCG.

AMORIM, L.V.; GOMES, C.M.; SILVA, F.L.H.; FERREIRA, H.C. Comportamento reológico de dispersões de argilas bentoníticas: efeitos do tipo de ferramenta, velocidade e tempo de agitação. Cerâmica. São Paulo, v. 48, n. 308, p. 234-238, 2002.

AMORIM, L.V.; PEREIRA, E. Bentonita da Paraíba: a exaustão de uma riqueza brasileira.
ABAS Informa. São Paulo, março, n. 147, p. 1617, 2003.

ASSUNÇÃO, L.M.C.; FERREIRA, H.C. Influência da concentração, velocidade e tempo de agitação nas propriedades reológicas de dispersões de montmorilonitas industrializadas no Estado da Paraíba. Cerâmica. São Paulo, v. 25, n. 114, p. 165-171, 1979.

BARROS NETO, B.; SCARMINIO, I.S.; BRUNS, R.E. Planejamento e otimização de experimentos, Editora da UNICAMP, São Paulo, 1996.

DARLEY, H.C.H.; GRAY, G.R. Composition and properties of drilling and completion fluids, 
Fifth Edition, Gulf Publishing Company, Houston, Texas, 1988.

MÉTODOS para análise química, Laboratório de Análises Minerais - Centro de Ciências e Tecnologia, Universidade Federal de Campina Grande, PB, 2000.

PETROBRAS, Viscosificante para fluido de perfuração base de água na exploração e produção de petróleo, Especificação, N-2604, 1998.

PETROBRAS, Ensaio de viscosificante para fluido de perfuração base de água na exploração e produção de petróleo, Método, N-2605, 1998a. SOUZA SANTOS, P. Ciência e tecnologia de argilas, Editora Edgard Blücher, São Paulo, v. 2, 1992.
STATSOFT, Inc. STATISTICA for Windows, version 5.0, 2000, $1 \mathrm{CD}$.

STEFAN, P. Métodos de ensaio de argilas e bentonitas para fluidos de perfuração. Cerâmica. São Paulo, v. 12, n. 47-48, p. 266, 1966.

VALENZUELA DÍAZ, F.R.; ABREU, L.D.V.; SOUZA SANTOS, P. Variação da viscosidade de misturas de argila-água com a concentração, Parte II. In: $36^{\circ}$ Congresso Brasileiro de Cerâmica, Caxambú, MG, v. 1, p.148-155, 1982. VALENZUELA DÍAZ, F.R.; ABREU, L.D.V.; SOUZA SANTOS, P. Variação da viscosidade de misturas de argila-água com a concentração. Cerâmica. São Paulo, v. 37, n. 248, p. 37-42, 1991. 
Estudo reológico de fluidos de perfuração à base de água: influência do teor de sólidos, velocidade e tempo de agitação 